\title{
Short communication: Volatiles in microfluidized raw and heat-treated milk
}

\author{
D. L. Van Hekken, S. landola, and P. M. Tomasula* \\ Dairy and Functional Foods Research Unit, Agricultural Research Service, USDA, Wyndmoor, PA 19038
}

\begin{abstract}
As innovative processing equipment is introduced to milk processing, it is essential to determine its effect on milk aroma, a critical factor in consumer acceptance of the final dairy product. Microfluidization is known to cause severe high-pressure homogenization of milk fat and, although severe processing is known to release undesired aromas, no information is available on the levels of the volatile compounds in milk immediately after microfluidization. We hypothesized that microfluidization would alter levels of volatile compounds in milk that may affect aroma. The concentration of 11 selected volatile compounds in raw, thermized, pasteurized, and UHT 3.0\% fat milk samples were compared before and after microfluidization at $170 \mathrm{MPa}$ and common 2-stage homogenization at $15 \mathrm{MPa}$. Overall, the different milk samples had similar trends in response to homogenization, although UHT milk started with lower values of nonanoic acid, and acetone and higher levels of hexanal and heptanol. In many cases, microfluidization did not significantly alter volatile levels compared with the starting milk. Heptanal was the only compound observed to increase in thermized and UHT milk, whereas nonanoic acid and acetone decreased in raw, thermized, and pasteurized milks and octanoic acid decreased in thermized and UHT milks. The highest levels of almost all of the volatiles were found in the 2-stage homogenized milk. Overall, microfluidization had minimal effect on the volatile compound profiles of milk, although sensory evaluation is needed to confirm effects on aroma and flavor.
\end{abstract}

Key words: milk, microfluidization, volatile compounds

\section{Short Communication}

As innovative equipment is evaluated for potential use in milk processing, it is essential to determine its

\footnotetext{
Received October 1, 2018.

Accepted June 4, 2019.

*Corresponding author: Peggy.Tomasula@ARS.USDA.gov
}

effect on milk aroma, a critical factor in consumer acceptance of the final dairy product. Microfluidization is known for its severe high-pressure homogenization of milk fat. Although severe processing is known to release undesired aromas, little information is available on the levels of volatile compounds in milk immediately after microfluidization.

Microfluidization, an innovative nonthermal processing technique, efficiently homogenizes the milk in one pass into an emulsion populated by submicron-sized fat droplets and components with altered protein-protein and protein-fat interactions. The major changes to the milk are induced when 2 streams of milk are forced under high pressure (up to $200 \mathrm{MPa}$ ) to collide from opposite directions within a reaction chamber, causing the components to be exposed to cavitation implosions that result in high temperature, pressure, and shear. Microfluidization has been used to improve the manufacture and texture of yogurt (Ian et al., 2011), but microfluidized milk forms denser, less flexible cheese matrices, which are detrimental to the texture of Cheddar and Mozzarella cheeses (Lemay et al., 1994; Van Hekken et al., 2007); however, no information is available on the volatile profiles of the milk immediately after microfluidization. The severe disruption of the fat droplets in milk is of concern because the fatty acid components are major contributors to the flavor of milk and highly susceptible to oxidative (aldehydes) and hydrolytic (free fatty acids) rancidity issues. Ultrahigh-pressure homogenization (UHPH), which uses a homogenization nozzle at pressures $>200 \mathrm{MPa}$, has been reported in a few studies to alter the aroma of milk (Amador-Espejo et al., 2014) and yogurts (Juan et al., 2009; Serra et al., 2009), and to alter proteolysis and lipolysis in aging cheeses (Zamora et al., 2015). Only a few studies have evaluated the volatile profile in UHPH milk (Pereda et al., 2008; Amador-Espejo et al., 2017). As new dairy food applications for microfluidized milk with altered functionalities are explored, the immediate effect of microfluidization on the volatile compound profile of the milk, a critical factor in consumer acceptance of the product, needs further elucidation.

The objectives of this study were to compare the levels of 11 selected volatile compounds in raw and heat- 
treated samples (thermized, pasteurized, and UHT) of $3.0 \%$ fat milk before and after microfluidization and 2 -stage homogenization and determine the degree of alteration after microfluidization.

The study was conducted in 2 phases, with experiments conducted in triplicate using a split-plot design with milk heat treatments as the whole plot and processing treatments as the sub-plot fixed factors. Data were analyzed using ANOVA Proc Mixed statements and Bonferroni mean comparison statements $(P<$ 0.05) (version 9.4; SAS Institute, Inc., Cary, NC). In the first phase, 3 shipments of 210 -L of raw milk were obtained from a local fluid processing plant, processed, and microfluidized in our Wyndmoor dairy pilot plant as described in Bucci et al. (2018). Briefly, for each shipment, milk was standardized to $3.0 \%$ fat, divided into 3 portions, and subjected to one of the following heat treatments: no heating; thermized heating $\left(65^{\circ} \mathrm{C}\right.$ for $\left.15 \mathrm{~s}\right)$; or $\mathrm{HTST}$ pasteurization $\left(75^{\circ} \mathrm{C}\right.$ for $15 \mathrm{~s}$ ) using either a Universal Pilot Plant (Waukesha Cherry-Burrell, Delavan, WI) or a UHT/HTST Heat Exchanger system (FT74XTS; Armfield Limited, Ringwood, UK). All milk was returned to $4^{\circ} \mathrm{C}$ storage. A 3 -L portion of each raw, thermized, and pasteurized milk was warmed to $45^{\circ} \mathrm{C}$ and homogenized $(10 \mathrm{MPa} / 5$ $\mathrm{MPa}$ ) in a standard 2-stage homogenizer (Niro Soavi NS2001 H; GE, Dusseldorf, Germany) as a second control. Within $48 \mathrm{~h}$ of delivery, 12-L portions of raw, thermized, and pasteurized milks were microfluidized (2 passes) at $54^{\circ} \mathrm{C}$ and $170 \mathrm{MPa}$. Samples for volatile compound evaluation were collected directly from the microfluidizer, rapidly chilled on ice, and immediately place in a $-40^{\circ} \mathrm{C}$ freezer until assayed. In the second phase, 3 shipments of $18 \mathrm{~kg}$ of raw milk were obtained and each shipment was standardized to $3.0 \%$ fat; then, a 1-L portion was removed for the no-heat control and the remaining milk was UHT pasteurized at $130^{\circ} \mathrm{C}$ for 2 s (M210-E/H Microfluidizer; Microfluidics Corp., Westwood, MA), and received the same homogenization and microfluidization treatments as in phase 1 .

Profiles of volatile compounds were obtained from frozen milk using a modified version of the method described by Tunick et al. (2015). Briefly, milk was thawed overnight at $4^{\circ} \mathrm{C}$ and triplicate $10-\mathrm{mL}$ aliquots were placed in clear glass $20-\mathrm{mL}$ vials with polytetrafluoroethylene silicon septum caps (Supelco, Bellefonte, PA), vortexed with $4 \mathrm{~g}$ of $\mathrm{NaCl}$ and an internal standard, $0.010 \mathrm{mg} / \mathrm{L}$ 2-methyl-3-heptanone (SigmaAldrich, St. Louis, MO), before being placed in an autosampler (CombiPAL; CTC Analytics, Zwingen, Switzerland). Each sample was warmed to $40^{\circ} \mathrm{C}$ and agitated at $500 \mathrm{rpm}$, and a divinylbenzene/carboxen/ polydimethylsiloxane (DVB/CAR/PDMS) solid-phase microextraction fiber (Supelco, Bellefonte, PA) adsorbed volatiles for $35 \mathrm{~min}$. Volatiles were separated on a GC-MS system (7890A GC and 59975C MS, Agilent Technologies, Santa Clara, CA, ) fitted with a DB-5 column (30-m, 0.25-mm i.d.; Restek Corp., Bellefonte, PA) and helium flowing at $0.6 \mathrm{~mL} / \mathrm{min}$. Volatiles were desorbed for $5 \mathrm{~min}$ in a splitless injector at $250^{\circ} \mathrm{C}$. The GC oven was held at $40^{\circ} \mathrm{C}$ for 2 min before increasing to $200^{\circ} \mathrm{C}$ at $10^{\circ} \mathrm{C} / \mathrm{min}$ and holding for $2 \mathrm{~min}$. Compounds were identified using the NIST library (NIST/ EPA/NIH Mass Spectra Library, version 2.0; https:// chemdata.nist.gov/) and comparison with known standards (Sigma-Aldrich). Integrated data were used to compare peak areas of volatiles with internal standards as described by Tunick et al. (2015).

Different processing steps, such as homogenization and heating, are known to alter the aromas and flavors of milk. Disruption of fat droplets by homogenization can lead to rancidity problems, and heating can enhance hydrolysis of milk components to form aroma and flavor compounds. Although 2-stage homogenization at $<25 \mathrm{MPa}$ is the standard for fluid milk processing, higher-pressure homogenization (>100 MPa) to obtain submicron fat emulsions is less common and typically reserved for value-added products. The different heat treatments selected in this study are used in the industry today: raw milk fresh from the farm; thermization (used to extend shelf life of raw milk that cannot be processed immediately); HTST pasteurization (used for most fluid milk products); and UHT pasteurization (used for shelf-stable fluid products). Heat causes a plethora of hydrolytic changes in the milk components, some desired and others not. The degree of change depends on the temperature and duration, with milder conditions having no or minimal effects and more severe exposures having direct and indirect effects on milk aroma and flavor (Hougaard et al., 2011; Dursun et al., 2017).

In this study, we focused on the volatiles that arise from milk fat because of the significant disruption caused by microfluidization. About $98 \%$ of the fatty acids are found in triacylglycerol form in milk and must be released for aroma and flavor compounds to be formed, either through lipase or processing stresses. Many of the key aromas and flavors in milk are derived from the short- (4- and 5-carbon chains) and medium-chain (6- to 10-carbon chains) fatty acids found in milk fat. The unsaturated fatty acids are transformed into aldehydes, acids, and alcohols, whereas the free fatty acids are transformed into esters. The 11 volatiles selected to evaluate the effect of microfluidization processing on raw, thermized, pasteurized, and UHT-treated milks were the only compounds consistently found in all milk 
samples evaluated and included 5 carboxylic acids, 3 aldehydes, 1 alcohol, and 2 ketones.

Carboxylic acids produced from short- and mediumchain fatty acids are associated with rancid or goaty aromas (Tunick, 2007). The 5 carboxylic acids measured in this study were butanoic, hexanoic, octanoic, nonanoic, and $n$-decanoic acids (Figure 1a, 1b, 1c, 1d, and $1 \mathrm{e}$, respectively). Compared with the levels found in control milk (black bars), microfluidization (gray bars) did not alter the amount of butanoic, hexanoic, or $n$-decanoic acids in any of the milk samples and decreased the levels of octanoic and nonanoic acids, although not always at significant levels. Nonanoic acid was the only acid compound that had lower levels $(P<0.05)$ in the control pasteurized and UHT milk samples compared with the raw and thermized milks. The carboxylic acids tended to be the highest in the 2-stage homogenized samples (white bars), especially in the raw and thermized milk, which reflects the presence of active lipase and unprotected fat substrates (milk fat globule membrane removed from fat droplets during homogenization). Although microfluidization removes the fat droplet membrane, the process also generates temperatures sufficient to inactivate lipases and alkaline phosphatase (Bucci et al., 2018).

Aldehydes derived from short-chain unsaturated fatty acids and from proteins (hydrolyzed by the Maillard reaction) are key volatiles in milk aroma and flavor, usually associated with fruity or green grass aromas (Tunick, 2007) as well as autoxidative rancid flavors. The 3 alkyl aldehydes measured were hexanal, heptanal, and nonanal (Figure 2a, 2b, and 2c, respectively). Levels of hexanal and heptanal were higher $(P<0.05)$ in UHT control (black bars) and microfluidized (gray bars) milk than in the other control milk samples. Hexanal concentrations were also higher $(P<0.05)$ in the 2 -stage homogenized raw and thermized milk samples. Heptanal was highest in the UHT samples. Concentrations of nonanal were not significantly different $(P>0.05)$ among any of the milk or processing treatments.

Alcohols are usually products of aldehydes and are associated with fruity aroma (Tunick, 2007). The only alcohol measured was 1-pentanol (Figure 2d). The UHT control milk had the highest $(P<0.05)$ levels followed by the 2-stage homogenized samples. In the raw, thermized, and pasteurized milks, levels of 1-pentanol before and after microfluidization were similar, whereas microfluidized samples had lower levels than the 2-stage homogenized samples.

Ketones are prevalent in milk and can have aromas that range from musty to green to fruity (Tunick, 2007). The 2 ketones measured were acetone and 2-butanone (Figure 2e and 2f, respectively). Acetone was the most prevalent of the 11 volatiles measured and decreased $(P$ $<0.05)$ following microfluidization in the raw, thermized, and pasteurized samples. The UHT milk contained about half the amount of acetone found in raw milk and the level did not change after microfluidization. The level of 2-butanone was similar in all milk samples and only in raw milk was there a significant decrease $(P$ $<0.05)$ following microfluidization.

Fresh milk is noted for having a bland flavor and a very slight aroma. Of the $400+$ volatiles identified in milk, 47 are classified as strong and 46 as moderate contributors to processed milk flavor (Nursten, 1997). Some volatiles require only minor increases in concentration to have a large effect on the aroma and flavor of milk because they are desired at low levels but considered unpleasant at higher levels. Some milk flavors and aromas require a mixture of compounds, many without detectable aromas, to create a desired smell or taste. Determining whether the changing volatile profile is correlated with detectable changes in the aromas and flavors of the processed milk requires sensory evaluation, preferably by trained panelists. In a product as bland as milk, any strong aroma will be quickly noticed. This may of be concern to manufacturers who use microfluidization on fluid milk or use it to emulsify other ingredients with milk and where they expect no flavor change in that step of the process.

A few studies have reported volatile profiles for milk super-homogenized using UHPH. This process forces milk under higher pressure (up to $400 \mathrm{MPa}$ ) through a small opening, whereas microfluidization uses the collision of 2 streams of milk under high pressure (up to $200 \mathrm{MPa}$ ) to homogenize milk; both processes reduce fat droplets to submicron sizes. Pereda et al. (2008) reported higher levels of hexanoic acid (associated with rancid flavors) and aldehydes in UHPH-treated milk. Amador-Espejo et al. $(2014,2017)$ reported that UHPH milk had lower cooked flavors and elevated aldehyde volatiles levels than UHT milk. Results from our study did not agree with these studies. The levels of hexanoic acid in the current study were not different $(P<0.05)$ among treatments, and unsaturated fatty acids tended to be lowest in the microfluidized samples. Some of the differences may be because of different inlet temperatures or differences in the mechanics of reducing fat droplet sizes.

Our microfluidized milk had higher levels of heptanal in thermized and UHT milks and tended to have similar hexanal and nonanal levels to raw milk. The levels of nonanoic acid and acetone decreased in raw, thermized, and pasteurized milks, whereas that of octanoic acid decreased in thermized and UHT milks. We also found that the common 2-stage homogenization samples typi- 
cally had the highest levels of carboxylic acids, hexanal, and pentanol in raw and thermized milks, although not always significantly higher. This may be because lipases were inactivated in pasteurized and microfluidized samples.

Overall, the different heat-treated milk samples had similar response to homogenization at low or high pressures, with higher levels being noted for the 2-stage homogenization for the carboxylic acids, hexanal, and pentanol. Microfluidized milk had volatile levels similar to or lower than raw milk, except for heptanal and pentanol in UHT milk. Bases on this limited study, microfluidization has a minimal effect on the volatile compound profile of milk, although further research should be conducted including more volatiles. Sensory research with trained panelists also is required to relate
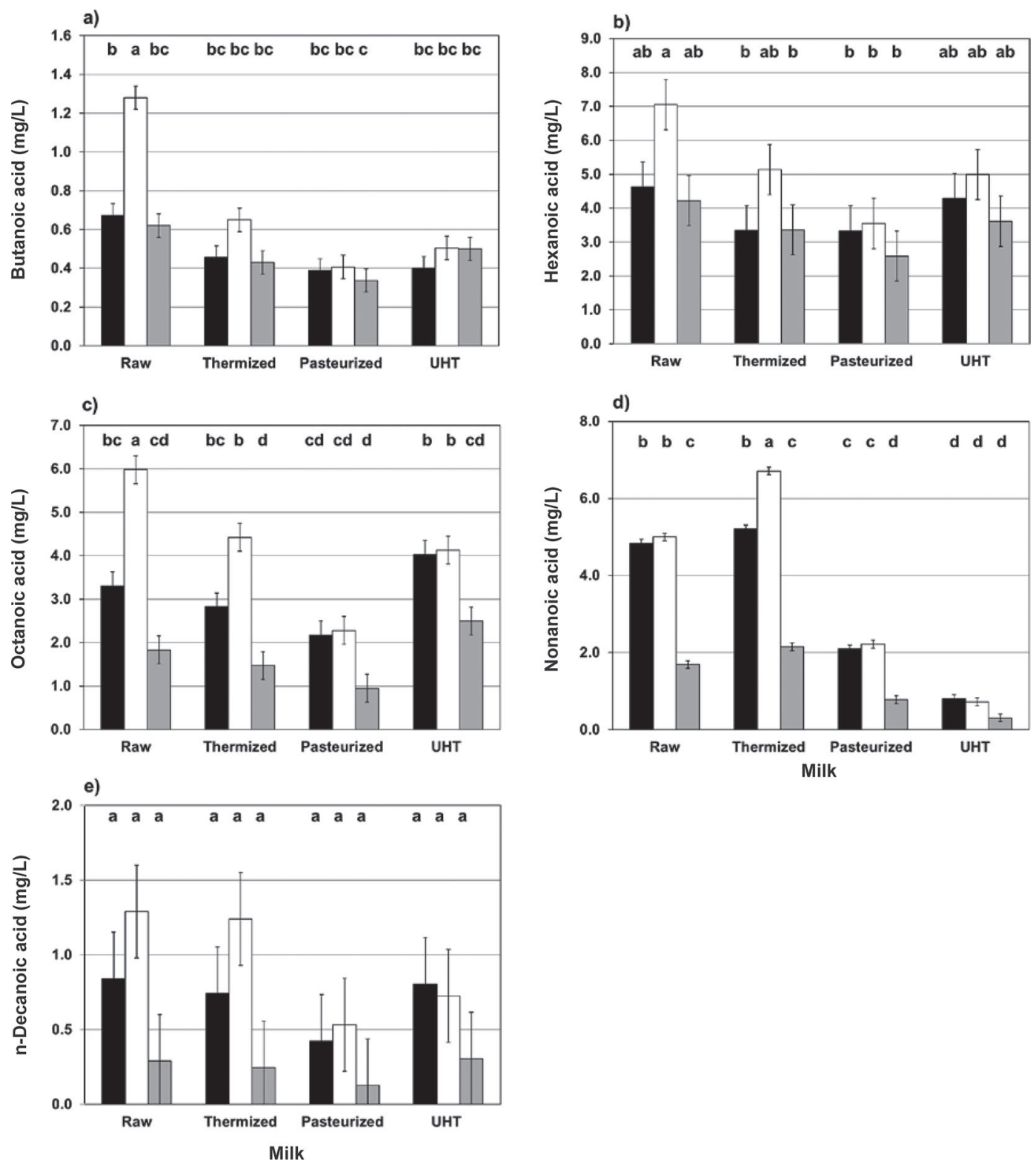

Figure 1. Levels of carboxylic acids (a) butanoic acid, (b) hexanoic acid, (c) octanoic acid, (d) nonanoic acid, and (e) n-decanoic acid present in raw and heat-treated (thermized, pasteurized, and UHT) milk that received one of the following treatments: controls with no further processing beyond heat treatment (black bars), 2-stage homogenization at $15 \mathrm{MPa}$ (white bars), and microfluidized homogenization at $170 \mathrm{MPa}$ (gray bars). Error bars represent SE; differing letters (a-d) above the histogram bars indicate significant differences, $P<0.05$. 

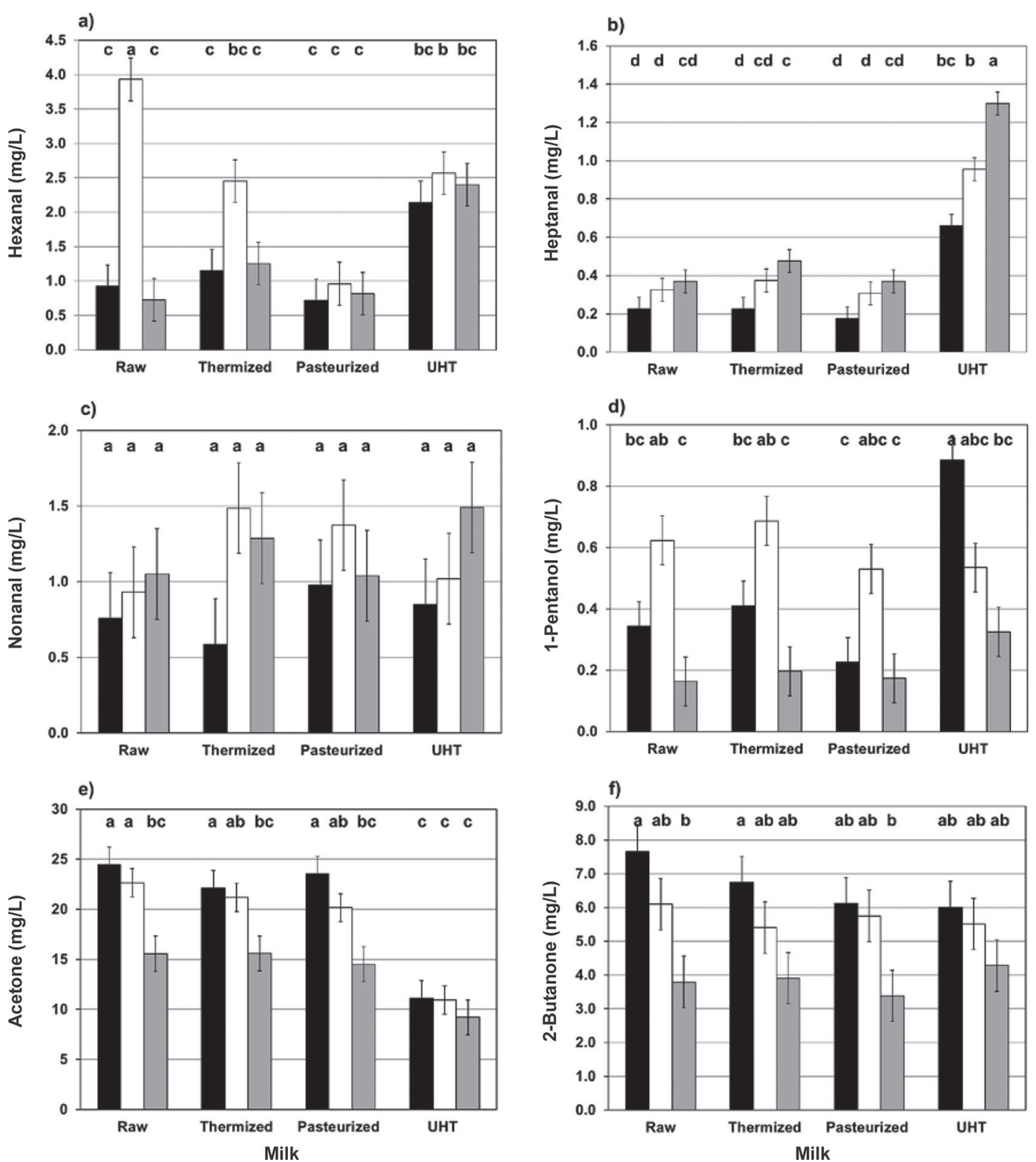

Figure 2. Levels of volatile aldehydes [(a) hexanal, (b) heptanal, (c) nonanal], alcohol [(d) 1-pentanol], and ketones [(e) acetone, (f) 2-butanone] present in raw and heat-treated (thermized, pasteurized, and UHT) milk that received one of the following treatments: controls with no further processing beyond heat treatment (black bars), 2-stage homogenization at $15 \mathrm{MPa}$ (white bars), and microfluidized homogenization at $170 \mathrm{MPa}$ (gray bars). Error bars represent SE; differing letters (a-d) above the histogram bars indicate significant differences, $P<0.05$.

the chemical profiles to the aromas and flavors actually detectable to humans and thus determine if microfluidization alters the sensory aspect of milk.

\section{ACKNOWLEDGMENTS}

Mention of commercial products in this publication is solely for the purpose of providing specific information and does not imply recommendation or endorsement by the US Department of Agriculture. The USDA is an equal opportunity provider and employer. The authors thank Agricultural Research Service researchers Ai Vi Truong and Ben Fulton for the help in preparing this manuscript.

\section{REFERENCES}

Amador-Espejo, G. G., A. Suàrez-Berencia, B. Juan, M. Bárcenas, and A. Trujillo. 2014. Effect of moderate inlet temperatures in ultrahigh-pressure homogenization treatments on physicochemical and 
sensory characteristics of milk. J. Dairy Sci. 97:659-671. https:// doi.org/10.3168/jds.2013-7245.

Amador-Espejo, G. G., J. J. Gallardo-Chacón, B. Juan, and A. J. Trujillo. 2017. Effect of ultra-high-pressure homogenization at moderate inlet temperatures on volatile profile of milk. J. Food Process Eng. 40. https://doi.org/10.1111/jfpe.12548.

Bucci, A. J., D. L. Van Hekken, M. H. Tunick, J. A. Renye, and P. M. Tomasula. 2018. The effects of microfluidization on the physical, microbial, chemical, and coagulation properties of milk. J. Dairy Sci. 101:6990-7001.

Dursun, A.. Z. Güler, and Y. E. Sekerl. 2017. Characterization of volatile compounds and organic acids in ultra-high temperature milk packaged in Tetra Brik cartons. Int. J. Food Prop. 20:1511-1521.

Hougaard, A. B., J. S. Vestergaard, C. Varming, W. L. P. Bredie, and R. H. Ipsen. 2011. Composition of volatile compounds in bovine milk heat treated by instant infusion pasteurization and their correlation to sensory analysis. Int. J. Dairy Technol. 64:34-44.

Ian, C., E. Ciron, V. L. Gee, A. L. Kelly, and M. A. E. Auty. 2011. Effect of microfluidization of heat-treated milk on rheology and sensory properties of reduced fat yoghurt. Food Hydrocoll. 25:14701476. https://doi.org/10.1016/j.foodhyd.2011.02.012.

Juan, B., J. M. Quevedo, B. Guamis, V. Ferragut, and A. J. Trujillo. 2009. Sensorial characteristics of goat milk cheeses made from ultra high-pressure homogenization-treated milk. Semantic Scholar, Athens, Greece. https://pdfs.semanticscholar.org/2b1a/ 0991bca8c49bac0e453c4aadfc52fcf349e2.pdf

Lemay, A., P. Paquin, and C. Lacroix. 1994. Influence of microfluidization of milk on Cheddar cheese composition, color, texture, and yield. J. Dairy Sci. 77:2870-2879.
Nursten, H. E. 1997. The flavor of milk and dairy products: I. Milk of different kinds, milk powder, butter and cream. Int. J. Dairy Technol. 50:48-56.

Pereda, J., D. P. Jarmillo, J. M. Quevedo, V. Ferragut, B. Guamis, and A. J. Trujillo. 2008. Characterization of volatile compounds in ultra-high-pressure homogenized milk. Int. Dairy J. 18:826-834.

Serra, M., A. J. Trujillo, B. Guamis, and V. Ferragut. 2009. Flavour profiles and survival of starter cultures of yoghurt produced from high-pressure homogenized milk. Int. Dairy J. 19:100-106. https:/ /doi.org/10.1016/j.idairyj.2008.08.002.

Tunick, M. H. 2007. Origins of Cheese Flavor. ACS Symposium Series, Vol. 971, Flavor of Dairy Products. American Chemical Society, Washington, DC

Tunick, M. H., M. Paul, E. R. Ingham, H. J. Karreman, and D. L. Van Hekken. 2015. Differences in milk characteristics between a cow herd transitioning to organic versus milk from a conventional dairy. Int. J. Dairy Technol. 68:511-518.

Van Hekken, D. L., M. H. Tunick, E. L. Malin, and V. H. Holsinger. 2007. Rheology and melt character of low fat and full fat Mozzarella cheese made from microfluidized milk. Lebensm. Wiss. Technol. 40:89-98. https://doi.org/10.1016/j.lwt2005.08.005.

Zamora, A., B. Juan, and A. J. Trujillo. 2015. Compositional and biochemical changes during cold storage of starter-free fresh cheeses made from ultra-high-pressure homogenized milk. Food Chem. 176:433-440. https://doi.org/10.1016/j.foodchem.2014.12.070. 